\title{
EDITORIAL
}

\section{A breakthrough in Hodgkin's disease}

recently had an opportunity to review an exciting paper by Steidl et al. ${ }^{1}$ published in the NEJM on 11 March 2010. Jose Costa and I wrote the accompanying editorial. ${ }^{2}$ I thought it would be worthwhile repeating some of our commentary for the journal's readership because I think it may be the data we have been looking for to change the direction of the treatment of Hodgkin's disease. Key to this study was the availability of fresh frozen tissue for gene profiling and additional paraffin fixed material for follow-up immunohistochemistry studies, all in populations of uniformly treated patients.

Steidl et al. ${ }^{1}$ discovered a gene signature of tumor associated macrophages and monocytes in patients with Hodgkin's disease that correlated with outcome. Remarkably, they were able to validate the correlation in an independent cohort of patients using CD68-an immunohistochemical marker of normal macrophages. The correlation of CD68 positive macrophages with outcome was striking. All patients with limited disease, minimally positive for CD68, were alive and free of disease at the time of the report. The association of CD68 positivity and disease-specific mortality rates was strong in all subsets analyzed. In advanced disease the correlation between macrophage number and progression-free survival is significant.

This study provides a rationale for the use of molecular tools to better understand cancers where effective treatments are available but cannot prospectively separate those who will not respond to current treatment from those who will be cured. Hodgkin's disease is a good example of this type of situation. For almost 40 years, early-stage disease has been curable by radiotherapy and combination chemotherapy can cure both early-stage and advanced-stage disease. Despite an overall cure rate of around $80 \%$, treatment has stagnated in the past two decades because of the absence of precise markers that can predict response to therapy. As a result of this situation most patients, especially those in early stages of disease, are over treated-they receive radiotherapy and combination chemotherapy. For example, in some studies where young women have received both chemotherapy and radiotherapy, the incidence of breast cancer reaches almost $30 \%$ at 15 years after treatment. ${ }^{3}$

Almost all patients with classic Hodgkin's disease will go into remission with initial treatment but about $30 \%$ of patients with advanced disease and almost $15 \%$ of those with early stages of disease will also relapse. Early relapses in patients with both advanced and localized disease treated with chemotherapy defines a drug resistance subset of Hodgkin's lymphoma. This group carries a very poor prognosis with them though all subsequent treatment approaches including high-dose treatment with stem cell support. ${ }^{4}$ It is of considerable interest that early relapse from complete remission carries the same poor prognosis in all tumor types where remission is possible, suggesting a common mechanism of resistance across tumor types. If at the time of diagnosis we could identify the small subset of Hodgkin's patients who are destined to fail to respond to chemotherapy, most patients could be spared the use of a combination of modalities as initial treatment, especially radiotherapy, which is associated with long-term toxicity.

The Reed-Sternberg (R-S) cell is unique among lymphoma cells in that a number of important signaling pathways have been shown to be constitutively activated, including the JAK-STAT pathway, receptor tyrosine kinases, NFKB and other pathways. ${ }^{5-7}$ The R-S cell also secretes numerous cytokines, including granulocytemacrophage colony-stimulating factor, which may be responsible for the assembly of inflammatory cells in involved lymph nodes. It has been termed the master regulator of the surrounding inflammatory response. ${ }^{5,7}$

Almost all tumors types are invaded by macrophages and it was once thought that this invasion represented a host immunological response to the tumor. However, most evidence now links tumor-associated macrophages (TAM) with a poor prognosis, ${ }^{8}$ as demonstrated in the study by Steidl and colleagues. Termed 'tropic macrophages' by Mantovani and Pollard,, ${ }^{9}, 10$ TAMs bear a close resemblance in function to embryonic macrophages associated with cell migration during development. ${ }^{11}$ These macrophages have been shown to mediate blood vessel formation by regulating the angiogenic switch through secretion of VEGF and hypoxia inducing factor. ${ }^{12}$

It is not difficult to imagine how an abundance of trophic macrophages would lead to tumor progression. It is somewhat more difficult to explain their impact on response to treatment. It seems likely that at some point in the evolution of the disease, a critical pathway to apoptosis is crippled in the R-S cell and this is associated with the secretion of a cytokine that leads to macrophage infiltration of the tumor. Such an event could inhibit cell death in response to cytotoxic treatments.

The data provided by Steidl et al. ${ }^{1}$ offer the breakthrough we have been looking for to select patients with a particularly poor prognosis, regardless of stage, for more-aggressive treatment and bring more logic to the treatment of this very curable malignancy.

doi:10.1038/nrclinonc.2010.36

Supplementary information is linked to the online version of the paper at http://www.nature.com/nrclinonc

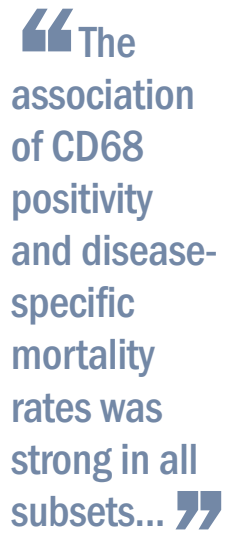

Vincent T. DeVita Jr is the Editor-in-Chief of Nature Reviews Clinical Oncology.

Competing interests The author declares no competing interests. 